\title{
PageRank for Bibliographic Networks
}

\author{
Dalibor Fiala $^{1,2}$, François Rousselot ${ }^{2}$, Karel Ježek ${ }^{1}$ \\ ${ }^{1}$ University of West Bohemia in Pilsen, Czech Republic \\ ${ }^{2}$ INSA Strasbourg, France
}

In this paper, we present several modifications of the classical PageRank formula adapted for bibliographic networks. Our versions of PageRank take into account not only the citation but also the co-authorship graph. We verify the viability of our algorithms by applying them to the data from the DBLP digital library and by comparing the resulting ranks of the winners of the ACM E. F. Codd Innovations Award. Rankings based on both the citation and co-authorship information turn out to be "better" than the standard PageRank ranking.

\section{Introduction}

Notions of importance, significance, authority, prestige, quality and others play a major role in social networks of all types. They denote an object that has a large impact on the other objects in the community. Perhaps the best example are bibliographic citations in the scientific literature. Counting citations of research publications is a relatively objective manner to determine quality research known since a long time ago. With the fast growth of the World Wide Web in the past ten years, this kind of analysis has become essential also in this domain in which links between Web pages may serve as citations. Therefore, current Web search engines make use of various link-based quality ranking algorithms whose rankings they combine with the keyword search

\section{Address for correspondence:}

Dalibor Fiala

Dept. of Computer Science and Engineering

University of West Bohemia in Pilsen

Univerzitní 22, 30614 Plzeň, Czech Republic

dalfia@kiv.zcu.cz 
results to offer the user not only topic-relevant but also high quality Web pages. The best-known link-based ranking algorithm is PageRank (BRIN, 1998). BIANCHINI (2005) and LANGVILLE (2003) review the latest developments of PageRank thoroughly. This recursive algorithm is applicable to any directed graph - such as a graph of citations between authors or papers. However, bibliographic data usually offers more than just citations. Collaboration networks are also a valuable source of information and are often studied (e.g. WAGNER, 2003, OTTE, 2002, CUNNINGHAM, 1997). But their combination with citation graphs, which may lead to more "fair" rankings of authors, has been relatively little examined. In the following sections, we present several modifications of the classical PageRank formula adapted for bibliographic networks. Our versions of PageRank take into account not only the citation but also the co-authorship information.

\section{Definitions}

Let $G^{P}=\left(P \cup A, E^{P}\right)$ be an undirected, unweighted, bipartite graph (coauthorship graph), $P \cup A$ a set of vertices ( $P$ a set of publications, $A$ a set of authors) and $E^{P}$ a set of edges. Each edge $\{p, a\} \in E^{P}, p \in P, a \in A$ means that author $a$ has (co)authored publication $p$. Let $G^{C}=\left(P, E^{C}\right)$ be a directed unweighted graph (publication citation graph), $P$ a set of vertices (the same set of publications), and $E^{C}$ a set of edges (citations between publications). Now, based on the two graphs $G^{P}$ and $G^{C}$, we will introduce yet another graph we will further work with. Let $G=(A, E)$ be a directed, edge-weighted graph (author citation graph), $A$ a set of vertices (the same set of authors) and $E$ a set of edges (citations between authors). For every $p \in P$ let $A_{p}=\{a \in A$ : $\left.\exists\{p, a\} \in E^{P}\right\}$ be the set of authors of publication $p$. For each $\left(a_{1}, a_{2}\right), a_{1} \in A, a_{2} \in A, a_{1} \neq a_{2}$ where there exists $\left(p_{1}, p_{2}\right) \in E^{C}$ such that $\left\{p_{1}, a_{1}\right\} \in E^{P}$ and $\left\{p_{2}, a_{2}\right\} \in E^{P}$ and $A_{p 1} \cap A_{p 2}=$ 
$\varnothing$ (i.e. no common authors in citing and cited publications are allowed) there is an edge $\left(a_{1}, a_{2}\right) \in E$. Thus, $\left(a_{1}, a_{2}\right) \in E$ if and only if $\exists\left(p_{1}, p_{2}\right) \in E^{C} \wedge \exists\left\{p_{1}, a_{1}\right\} \in E^{P} \wedge \exists\left\{p_{2}, a_{2}\right\} \in E^{P}$ $\wedge A_{p 1} \cap A_{p 2}=\varnothing \wedge a_{1} \neq a_{2}$.

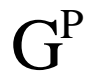

$\mathrm{p}_{1}$

$\mathrm{p}_{2}$

$\mathrm{p}_{3}$

$\mathrm{p}_{4}$

$\mathrm{G}^{\mathrm{P}}$<smiles>[AlH2]</smiles>

(1)

(1)

(1)

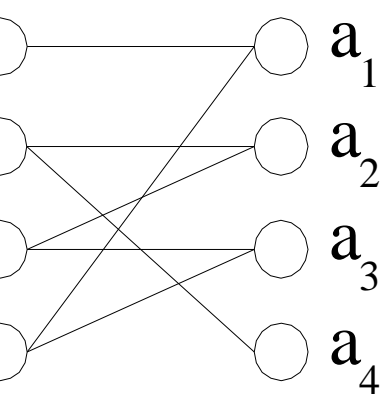

$\mathrm{G}^{\mathrm{C}}$

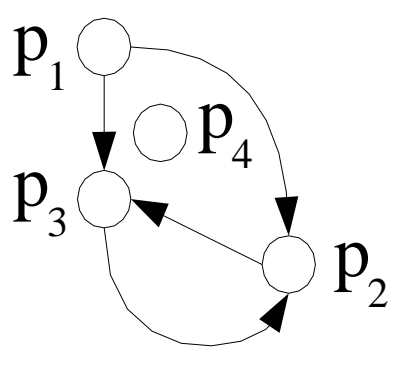

G

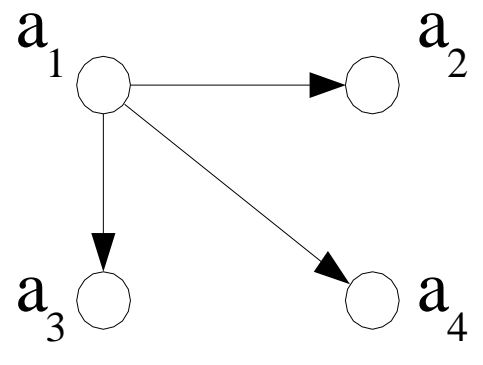

Figure 1: Examples of co-authorship, publication citation, and author citation graphs.

Before assigning weights to edges in $E$, we further define:

- $w_{u, v}=|C|$ where $C=\left\{p_{1} \in P: \exists\left\{p_{1}, u\right\} \in E^{P} \wedge \exists\left\{p_{2}, v\right\} \in E^{P} \wedge \exists\left\{p_{1}, p_{2}\right\} \in E^{C} \wedge p_{1} \neq\right.$ $\left.p_{2}\right\}$, as the number of citations from $u$ to $v$,

- $f_{u, v}=\left|P_{u}\right|+\left|P_{v}\right|$ where $P_{i}=\left\{p \in P: \exists\{p, i\} \in E^{P}\right\}$, as the number of publications by $u$ plus the number of publications by $v$,

- $c_{u, v}=|C P|$ where $C P=\left\{p \in P: \exists\{p, u\} \in E^{P} \wedge \exists\{p, v\} \in E^{P}\right\}$, as the number of common publications by $u$ and $v$,

- $h d_{u, v}=\left|A D C_{u}\right|+\left|A D C_{v}\right|$ where $A D C_{i}=\left\{\mathrm{a} \in A: \exists \mathrm{p} \in P\right.$ such that $\{p, a\} \in E^{P} \wedge$ $\left.\{p, i\} \in E^{P}\right\}$, as the number of all distinct co-authors of $u$ plus the number of all distinct co-authors of $v$,

- $h_{u, v}=\left|A D C_{u}\right|+\left|A D C_{v}\right|$ where $A D C_{i}$ is defined as above but it is a multiset, as the number of all co-authors of $u$ plus the number of all co-authors of $v$, 
- $t d_{u, v}=|D C A|$ where $D C A=\left\{a \in A: \exists \mathrm{p} \in P\right.$ such that $\{p, a\} \in E^{P} \wedge\{p, u\} \in E^{P} \wedge$ $\left.\{p, v\} \in E^{P}\right\}$, as the number of distinct co-authors in common publications by $u$ and $v$,

- $t_{u, v}=|D C A|$ where $D C A$ is defined as above but it is a multiset, as the number of co-authors in common publications by $u$ and $v$,

- $g_{u, v}=f_{u, v}-\left|S P_{u}\right|-\left|S P_{v}\right|$ where $S P_{i}=\left\{p \in P:\{p, i\} \in E^{P} \wedge d_{G^{P}}(p)=1\right\}$, as the number of publications by $u$ where $u$ is not the only author plus the number of publications by $v$ where $v$ is not the only author.

Note that the current authors are considered as co-authors of themselves (variables $h d, h, t d, t$ ). They should actually not be counted in, but this would have no effect on the results.

\section{Rank Computation}

We associate a triple of weights $\left(w_{u, v}, c_{u, v}, b_{u, v}\right)$ with each edge $(u, v) \in E . w_{u, v}$ and $c_{u, v}$ are described above, and $b_{u, v}$ can be equal to one of the seven following values according to the semantics of edge weights we want to stress: a) zero, b) $f_{u, v}$, c) $h_{u, v}$, d) $h d_{u, v}$, e) $g_{u, v}$, f) $t_{u, v}$, g) $t d_{u, v}$. We then define the rank $R(u)$ for author $u$ as follows:

$$
R(u)=\frac{1-d}{|A|}+d \sum_{(v, u) \in E} R(v) \frac{\sigma_{v, u}}{\sum_{(v, k) \in E} \sigma_{v, k}}
$$

where

$$
\sigma_{v, k}=\frac{w_{v, k}}{\frac{c_{v, k}+1}{b_{v, k}+1} \sum_{(v, j) \in E} w_{v, j}}
$$

and $d$ is the damping factor, an empirically determined constant usually set to about 0.9 . 
In all the variations above, we penalize the cited author for the frequency of collaboration with the citing author. We suppose that a citation obtained from a frequent co-author (colleague) is less valuable than that from a foreign researcher. Therefore, the contribution from citing authors is inversely proportional to the number of common publications with the cited author. This happens in case a). On the other hand, we mitigate this penalization under some circumstances. In cases c), d), f), and g) we recognize that the relationship between two authors is weaker if they have many coauthors in general - cases c) and d) - or in common publications - cases f) and g). We also distinguish between all co-authors - cases c) and f) - and distinct co-authors - cases d) and g). In case b) we claim that two authors are more closely related if they have relatively many common publications in relation to the total number of publications by both of them and less related in the opposite case. The same holds for case e) where the total number of publications by each author as the only author is counted. When all the coefficients $c$ and $b$ are equal to zero, equation (1) becomes the weighted PageRank formula. (For instance, BOLLEN (2006) and XING (2004) work with weighted PageRanks.) In addition to this, if all the weights $\mathrm{w}_{\mathrm{u}, \mathrm{v}}$ are set to one, it is the standard PageRank (BRIN, 1998). The coefficients $c$ and $b$ are analogous to the co-authorship frequency and exclusivity in (LIU, 2005) as noted on the related work.

\section{Zero $c$ coefficients}

Certainly, there will be many author pairs in $G$ for which $c$ is zero. Does it make sense to have a non-zero coefficient $b$ if $c$ is equal to zero? It surely does not when $b$ is $t$ or $t d$. If there are no common publications, there are no co-authors in common publications either. Other parameters $(f, g, h, h d)$ may (or even must) be greater than zero even if $c$ is zero. But modifying the portion of rank distributed between authors 
only on the basis of all their publications $(f)$, all their co-authors $(h)$, etc. without the context of their common publications $(c=0)$ does not look meaningful. Why should author $x$ obtain more rank than author $y$ from a particular citing author only for the reason that he/she has written more publications? Briefly, we set $b$ to zero whenever $c$ is zero.

\section{Example}

Table 1 shows edge weights for graph $G$ in Figure 1 . The coefficients $f, g, h$, and $h d$ are zero when $c$ is zero as mentioned in the paragraph above, but their non-zero variants are also presented in parentheses for illustration. Edges $\left(p_{2}, p_{3}\right)$ and $\left(p_{3}, p_{2}\right)$ have no effect, because they are considered as self-citations (author $a_{2}$ has co-authored both of them). The proportions of rank distributed by author $a_{l}$ in graph $G$ in Figure 1 along its out-edges in the standard (PR) and weighted PageRank (w) and the variations a) - g) are given in Table 2 .

Table 1: Edge weights for graph $G$ in Figure 1.

\begin{tabular}{|c|c|c|c|c|c|c|c|c|}
\hline Edge & $\boldsymbol{w}$ & $\boldsymbol{c}$ & $\boldsymbol{f}$ & $\boldsymbol{g}$ & $\boldsymbol{h}$ & $\boldsymbol{h d}$ & $\boldsymbol{t}$ & $\boldsymbol{t d}$ \\
\hline$\left\{a_{1}, a_{2}\right\}$ & 2 & 0 & $0(4)$ & $0(1)$ & $0(7)$ & $0(4)$ & 0 & 0 \\
\hline$\left\{a_{1}, a_{3}\right\}$ & 1 & 1 & 4 & 1 & 7 & 3 & 2 & 2 \\
\hline$\left\{a_{1}, a_{4}\right\}$ & 1 & 0 & $0(3)$ & $0(1)$ & $0(5)$ & $0(4)$ & 0 & 0 \\
\hline
\end{tabular}

Table 2: Proportions of rank distributed by node $\mathrm{a}_{1}$ in graph $G$ in Figure 1.

\begin{tabular}{|l|c|c|c|c|c|c|c|c|c|}
\hline Edge & PR & $\mathbf{w}$ & $\mathbf{a}$ & $\mathbf{b}$ & $\mathbf{c}$ & $\mathbf{d}$ & $\mathbf{e}$ & $\mathbf{f}$ & $\mathbf{g}$ \\
\hline$\left\{\mathrm{a}_{1}, \mathrm{a}_{2}\right\}$ & $1 / 3$ & $2 / 4$ & $4 / 7$ & $4 / 11$ & $2 / 7$ & $2 / 5$ & $2 / 4$ & $4 / 9$ & $4 / 9$ \\
\hline$\left\{\mathrm{a}_{1}, \mathrm{a}_{3}\right\}$ & $1 / 3$ & $1 / 4$ & $1 / 7$ & $5 / 11$ & $4 / 7$ & $2 / 5$ & $1 / 4$ & $3 / 9$ & $3 / 9$ \\
\hline$\left\{\mathrm{a}_{1}, \mathrm{a}_{4}\right\}$ & $1 / 3$ & $1 / 4$ & $2 / 7$ & $2 / 11$ & $1 / 7$ & $1 / 5$ & $1 / 4$ & $2 / 9$ & $2 / 9$ \\
\hline$\sum$ & 1 & 1 & 1 & 1 & 1 & 1 & 1 & 1 & 1 \\
\hline
\end{tabular}

For example, to compute $\sigma_{a 1, a 2}$ for the variation w), we substitute in (2);

$$
\sigma_{a 1, a 2}=\frac{2}{\frac{0+1}{0+1}(2+1+1)}
$$


which is $2 / 4$. Since $\sigma_{a 1, a 2}+\sigma_{a 1, a 3}+\sigma_{a 1, a 4}=2 / 4+1 / 4+1 / 4=1$, the proportion $\frac{\sigma_{a 1, a 2}}{\sum_{(v, k) \in E} \sigma_{v, k}}$ from (1) remains 2/4. Thus, one half of rank of author $a_{1}$ is transferred to author $a_{2}$ and so on.

\section{Experiments}

We tested our algorithms on the DBLP data available in XML (http://dblp.unitrier.de/xml/). DBLP has established itself as a testbed for bibliographic studies in recent years (e.g. ELMACIOGLU, 2005, RAHM, 2005, BALMIN, 2004, or NASCIMENTO, 2003). We took advantage of the only time-stamped version of the collection from February 14, 2004 which may serve researchers as a testbed for experiments and comparisons. We extracted only article and inproceedings records exactly like SIDIROPOULOS $(2005,2006)$.

\section{DBLP Testbed Data}

Table 3 summarizes some basic statistics of the DBLP data we work with. We spend some time discussing it here as a good understanding of it is vital for everyone wishing to reproduce our experiments. The data contained 173630 article records (journal papers) and 298413 inproceedings records (conference papers) that we imported into a relational database. These numbers are in cells B2 and C2, respectively. The total number of article and inproceedings records (i.e. their corresponding XML elements), which we will refer to as papers, is 472043 (D2). The number of papers having some references is only 8188 (D3) which is less than two percent of the total. In addition, a large part of all references from papers (D6) are references to undisclosed publications outside of the DBLP library. The references within DBLP (D7) can be further decomposed into references to papers (D8) and references to other kinds of 
publications such as books, theses, etc. The corresponding numbers of papers with references within DBLP publications and with references to papers are D4 and D5. Exactly 18285 distinct papers are cited (D11). Time spans are not shown in Table 3. However, the most recent paper is from 2004, the oldest one is from 1936. The time period of citing papers is 1970 - 2001, that of cited papers is 1945 - 2001 We can also obtain other information from Table 3, such as the number of references from journal papers to conference papers (B10), the number of conference-to-conference references (C10), the number of journal papers with references to papers (B5), etc.

Table 3: Statistics of article and inproceedings records in DBLP 14 Feb 2004.

\begin{tabular}{rc|rrr} 
& A & \multicolumn{1}{c}{ B } & \multicolumn{1}{c}{ C } & D \\
1 & & articles & inproceedings & \multicolumn{1}{c}{ total } \\
\hline 2 & \# & 173630 & 298413 & 472043 \\
3 & \# with ref. & 1818 & 6370 & 8188 \\
4 & \# with ref. within DBLP & 1791 & 6212 & 8003 \\
5 & \# with ref. to papers & 1771 & 6177 & 7948 \\
6 & \# references & 47329 & 120822 & 168151 \\
7 & \# ref. within DBLP & 30186 & 79003 & 109189 \\
8 & \# ref. to papers & 27801 & 72853 & 100654 \\
\cline { 3 - 5 } 9 & \# ref. to articles & 13330 & 29247 & 42577 \\
10 & \# ref. to inproc. & 14471 & 43606 & 58077 \\
11 & \# distinct cited & 7391 & 10894 & 18285
\end{tabular}

\section{Publications}

Let us return to Table 3. The publication citation graph $G^{C}$ based on the articles and inproceedings records will thus have 472043 nodes $(|\mathrm{P}|$ in $\mathrm{D} 2)$ and 100654 edges $\left(\left|E^{C}\right|\right.$ in D8). So the references not pointing to papers or even pointing outside of DBLP have absolutely no effect. 7948 nodes (D5) will have some out-edges and 18285 nodes (D11) will have some in-edges. There will be 5389 nodes with both in- and outdegree non-zero (not shown in Table 3). The other graph constructed from the DBLP records is the co-authorship graph $G^{P}$. This graph has $|P|+|A|$ nodes (publications plus authors) which is $472043+315485=787528$ vertices in total. The number of edges 
$\left|E^{P}\right|$ is 1070643 . This is actually the number of publication - author pairs (see $G^{P}$ in Figure 1). The most frequent number of co-authors is two and a publication has 2.27 coauthors on average. Interestingly, there are also publications without any authors which is an obvious omission in DBLP.

\section{Author citation graph}

The resulting citation graph of authors $G$ had 295531 edges (no self-citations are allowed and citations between publications that have at least one common author are considered as self-citations) which is $|E|$. Obviously, $|A|$ is still 315485.12934 nodes had a non-zero in-degree, 6992 nodes had a non-zero out-degree. 4748 nodes had both a non-zero in-degree and a non-zero out-degree. Only 15178 authors were not isolated. This low inter-linkage of nodes in $G$ is a result of the nature of the DBLP data. Citations were systematically input only for a small number of journals and conferences, such as SIGMOD Record or VLDB Journal, as was already mentioned by SIDIROPOULOS (2005). See Figure 2 for a cumulative distribution of in- and out-degrees and their weighted variations (citations and references) in graph $G$.

The maximum value for in-degree is 1857 , for out-degree 834 , for citations (in) 5346 and for references (out) 2 594. Apparently, the largest bin would be 0+ (in-degree or citations of zero or more) with all the isolated authors included. It is not depicted in Figure 2. As we may see, the four series are quite well correlated. The number of authors with a specific degree decreases as the degree gets bigger. There are no evident outliers. Perhaps the most interesting feature is the sudden drop in the number of authors for $1+$ (having one or more) and $5+$ (having five or more) in-degree and citations. This is not the case for out-degree or references. This means that 5 is quite a boundary for less and more cited authors. Also, the superiority of references over 
citations which begins with $10+$ and terminates with $200+$ indicates that the group of highly cited authors is greater than that of highly citing authors.

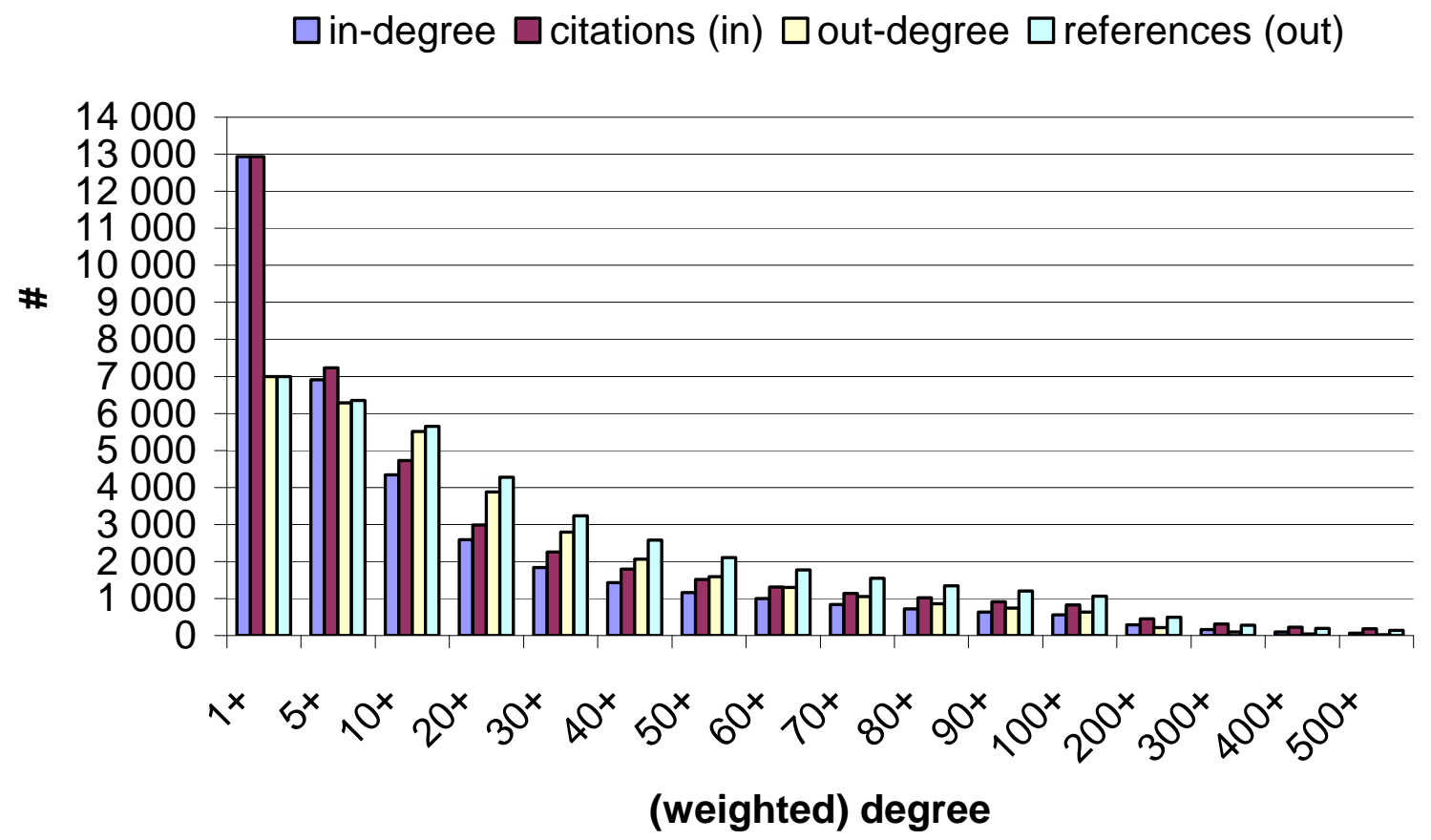

Figure 2: Cumulative histogram showing distribution of in- and out-degrees in $G$.

\section{Distribution of $c$ and $b$ coefficients}

Figure 3 shows the cumulative distributions of various parameters defined in the weights of edges in $E$ of graph $G$. The size of the bin $0+$ for each series of each graph would be 295531 , i.e. $|E|$. The number of edges in each $1+$ bin is always 7017 since this is the number of edges in $E$ between authors that have some common publications. This number will never be exceeded by values of other parameters because we have defined the parameters $f, g, h, h d, t, t d$ to be zero whenever $c$ is zero. Now, let us make a few examples of interpretation of the data in the figures. For instance, the number of edges in $E$ for which the parameter $c$ is five or more is a little greater than one thousand. This means that there are some one thousand author pairs having five common publications at least that cite each other (not necessarily at the same time). The author 
pairs are ordered, so if the authors cite one another at the same time, i.e. there are two edges in $E$ for this pair, the pair is counted twice. Another example: there are some 5000 author pairs having some common publications whose sum of publications is 70 at least (see Figure 3 top right).
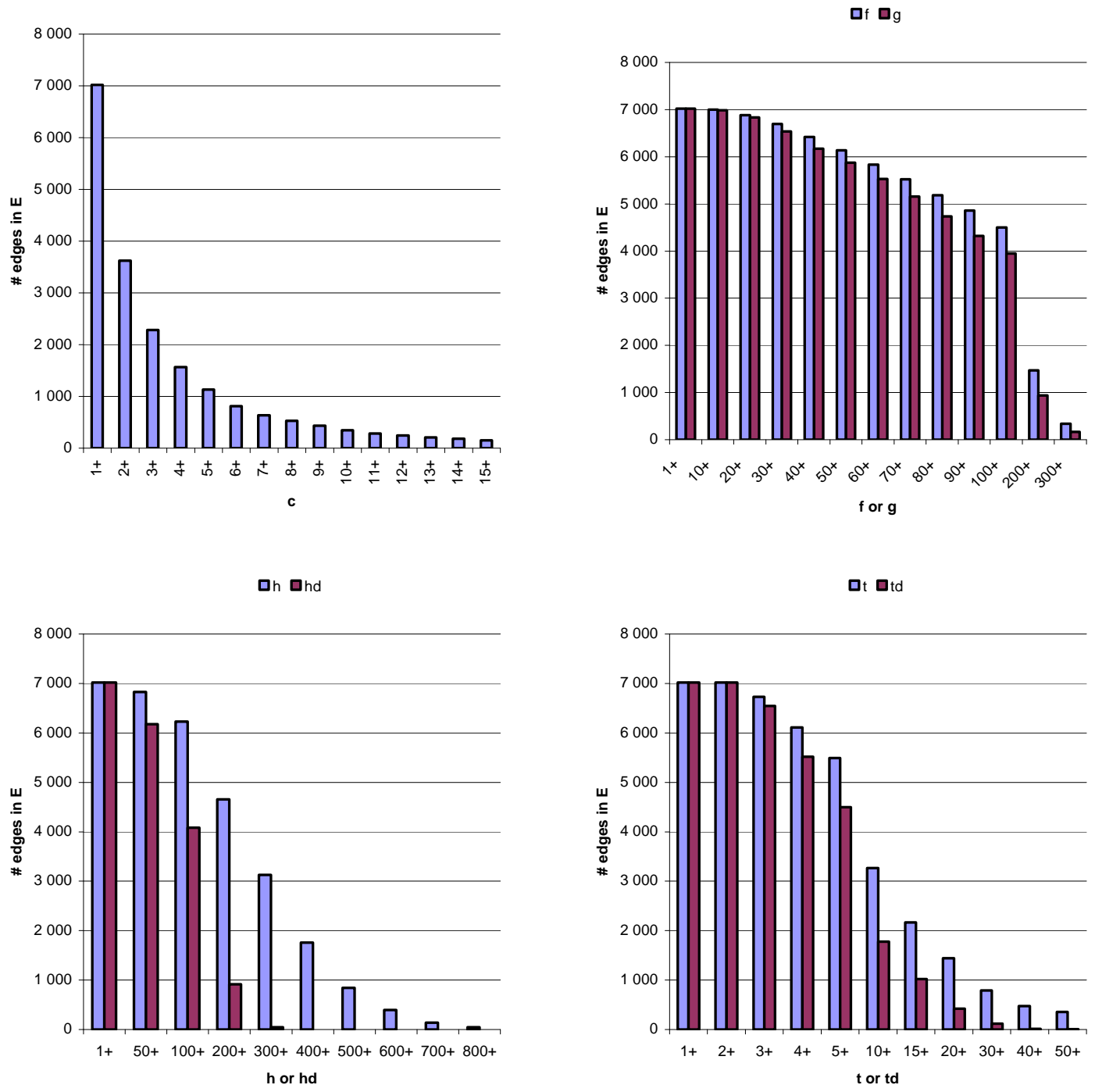

Figure 3: Cumulative distribution of values of parameters $c$ (top left), $f, g$ (top right), $h$, $h d$ (bottom left) and $t, t d$ (bottom right) in graph $G$..

In Figure 3 bottom left, we can observe that there are no collaborating authors that would have 400 or more distinct co-authors in total. The bins $1+$ and $2+$ in Figure 3 bottom right are the same because each common publication of two authors has two 
(distinct) co-authors at least. The largest number of author pairs have between five and ten distinct co-authors in their common publications (see Figure 3 bottom right). If we subtract the citing and the cited author, it is between three and eight. In general, it holds that $f \geq g, h \geq h d, t \geq t d$ as the second parameter in the couple is always more restrictive.

\section{Statistics of $c$ and $b$ coefficients}

To terminate this subsection, Table 4 presents basic statistics of the $c$ and $b$ parameters in the weights of edges in graph $G$, which were commented on in the previous paragraphs. Parameter $b$ is represented by the corresponding coefficients $f, g$, $h, h d, t$, and $t d$ as described in the definitions. Note that only those edges in $E$ of $G$ are considered for which $c$ is non-zero, i.e. edges between authors who have some common publications. The number of these edges is 7017 as mentioned above. Taking into account all of the edges in $E$ would obviously decrease the mean values and set all medians and modes to zero. In total, we have found 10902 author pairs having one common publication at least but not all of them have a citation edge in $E$, of course. Some interesting findings visible in Table 4 include: i) the maximum number of distinct co-authors in common publications by two specific authors is 67 (!), ii) the most frequent number of the same is three (rather low), iii) the maximum total number of publications (counted separately) of two collaborating authors is 489, etc. Much more analysis (such as component analysis) of the co-authorship and citations graphs could be done, but it is not the aim of this paper.

Table 4: Basic statistics of weight parameters for edges in $E$ with non-zero $c$.

\begin{tabular}{l|rrrrrrrr} 
& \multicolumn{1}{c}{$\boldsymbol{c}$} & \multicolumn{1}{c}{$\boldsymbol{f}$} & $\boldsymbol{g}$ & $\boldsymbol{h}$ & $\boldsymbol{h} \boldsymbol{d}$ & $\boldsymbol{t}$ & $\boldsymbol{t} \boldsymbol{d}$ \\
\hline $\boldsymbol{m i n}$ & 1 & 4 & 2 & 2 & 2 & 2 & 2 \\
$\max$ & 56 & 489 & 443 & 977 & 355 & 210 & 67 \\
avg & 2.93 & 139.83 & 120.87 & 295.26 & 122.41 & 14.80 & 7.99 \\
std. deviation & 3.89 & 81.50 & 72.28 & 168.68 & 64.50 & 17.66 & 6.47 \\
median & 2 & 130 & 111 & 273 & 114 & 9 & 6 \\
mode & 1 & 153 & 134 & 188 & 59 & 3 & 3
\end{tabular}




\section{Computing Ranks for Authors}

We exploited extensively the author citation graph $G$ described in detail above. Altogether, twelve ranking methods were employed to evaluate the authors. In addition to the weighted (citation counting) and unweighted in-degree, HITS authorities, and the standard (unweighted) PageRank, we also applied the weighted and the bibliographic (seven variants) PageRank algorithms. In this way, we finally obtained twelve author rankings. The big problem that immediately arises is how to evaluate the quality of these rankings. The quality of a ranking is a highly subjective matter. A straightforward solution would be to compare the generated rankings with an official, "human-made" ranking. Unfortunately, this does not exist. Another possibility would be to make use of the various available citation systems and compare the new rankings with their citationbased rankings. The trouble here is that the citation data in DBLP is very incomplete and it is more or less concentrated on publications in a few particular journals and conferences. Thus, it would not be directly comparable.

\section{Awards}

It is remarkable in this context, that ACM SIGMOD Digital Review and ACM SIGMOD Record journals as well as the ACM SIGMOD Conference have their publications' citations included. This was perhaps what initially triggered the idea in (SIDIROPOULOS, 2005) - namely to compare author rankings with lists of ACM SIGMOD award winners. Quite logically, the authors expected that award winners should be placed higher in their rankings than other authors. In other words, the "better" a ranking, the higher ranks it associates with award winning authors. As our approach is somewhat different from theirs (more on this will be said in the related work section), the only award we can take advantage of is the ACM SIGMOD E. F. Codd Innovations 
Award (http://www.sigmod.org/sigmodinfo/awards/\#innovations), which is awarded "for innovative and highly significant contributions of enduring value to the development, understanding, or use of database systems and databases."

\section{Program committees}

The only alternative approach to author ranking evaluation we are aware of is described by LIU (2005). Here the newly derived rankings are compared to lists of program committee members (i.e. prestigious researchers) of conferences on digital libraries. A ranking with more authors being members of program committees is considered "better" than another one having only a few of them. This approach has two obvious drawbacks. First, it is domain specific. It is appropriate for rankings based on data from digital library conferences (as was the case). For other fields different program committees would have to be considered. But for general, non-specific data (more or less the case of DBLP) it is not reasonable. And second, actual ranks of authors are not taken account of. So two rankings with the same authors in a different order would be evaluated the same. (Although this can be improved easily by comparing a series of ranks rather than single total scores.)

\section{Results}

We thus compared the ranks achieved by fifteen winners of the ACM SIGMOD E. F. Codd Innovations Award from the years 1992 - 2006. We also expected that "better" rankings would place award winners higher. Let us have a look at Table 5 with the actual ranks. The first three rankings (citations, in-degree and HITS authorities) are presented just for reference. The actual baseline ranking is "PR" (standard unweighted PageRank, in a darker column). In other words, the goal is to compare the new "bibliographic" PageRank rankings in columns "w" and "a" through "g" with the 
standard PageRank. The column "w" stands for the weighted PageRank and "a" - "g" correspond to the variations a) $-\mathrm{g}$ ) mentioned at the very beginning of the section on rank computation. We can see that the weighted PageRank is much better than the classical one in terms of the sum of ranks (the smaller the better), the median rank and a little better as for the worst rank assigned to the award winners. The rankings "a" - "g" are always better than the standard PR regarding the sum of ranks and median rank and only "a" and "c" have a worse worst rank. The ranking "a" is also weaker than "w" in all metrics whereas "c" only with respect to the worst rank. The rankings " $d$ " and "e" are the best in the sum of ranks and in the worst rank respectively. The median is better for "d" (9 versus 12). Let us recall that this ranking penalizes authors frequently cited by their co-authors but it weakens this handicap if the citing and cited authors have many distinct co-authors altogether. Moreover, the median rank 9 is the best of all in the table. Even the rankings not based on PageRank are worse in this respect.

Table 5: E. F. Codd Innovations Award winners and their ranks in distinct methods.

\begin{tabular}{|c|c|c|c|c|c|c|c|c|c|c|c|c|}
\hline Author & Cites & Deg & IITS & PR & w & $\mathbf{a}$ & b & c & d & e & f & g \\
\hline 1992 Michael Stonebraker & 1 & 1 & 1 & 3 & 2 & 2 & 1 & 1 & 1 & 1 & 3 & 3 \\
\hline 1993 Jim Gray & 4 & 3 & 4 & 6 & 3 & 6 & 2 & 2 & 2 & 4 & 1 & \\
\hline 1994 Philip Bernstein & 6 & 8 & 7 & 4 & 6 & 5 & 6 & 6 & 4 & 6 & 5 & \\
\hline 1995 David DeWitt & 2 & 2 & 2 & 36 & 14 & 20 & 3 & 3 & 3 & 2 & 4 & \\
\hline 1996 C. Mohan & 36 & 47 & 45 & 113 & 110 & 116 & 62 & 59 & 65 & 65 & 105 & 101 \\
\hline $1997 \mathrm{Da}$ & 13 & 11 & 11 & 51 & 35 & 47 & 7 & 7 & 6 & 7 & 11 & 13 \\
\hline $1998 \mathrm{Se}$ & 12 & 18 & 21 & 104 & 61 & 69 & 12 & 11 & 14 & 12 & 37 & 43 \\
\hline $1999 \mathrm{Hec}$ & 9 & 12 & 18 & 60 & 49 & 62 & 4 & 4 & 5 & 3 & 16 & 14 \\
\hline $2000 \mathrm{Ra}$ & 11 & 15 & 25 & 65 & 58 & 64 & 16 & 19 & 18 & 15 & 49 & 49 \\
\hline 2001 Rudolf Bayer & 84 & 75 & 94 & 7 & 16 & 14 & 97 & 132 & 94 & 93 & 25 & 20 \\
\hline 2002 Patricia Selinger & 38 & 38 & 23 & 59 & 55 & 53 & 61 & 55 & 54 & 63 & 36 & 48 \\
\hline 2003 Don Chamberlin & 16 & 13 & 10 & 2 & 4 & 3 & 29 & 26 & 23 & 26 & 7 & 6 \\
\hline 2004 Ronald Fagin & 28 & 40 & 46 & 19 & 13 & 13 & 27 & 28 & 30 & 25 & 17 & 17 \\
\hline 2005 Michael Carey & 7 & 9 & 5 & 63 & 46 & 55 & 13 & 10 & 9 & 14 & 21 & 29 \\
\hline 2006 Jeffrey D. Ullman & 3 & 5 & 9 & 15 & 8 & 12 & 5 & 5 & 7 & 5 & 8 & 8 \\
\hline Worst rank & 84 & 75 & 94 & 113 & 110 & 116 & 97 & 132 & 94 & 93 & 105 & 101 \\
\hline Sum of ranks & 270 & 297 & 321 & 720 & 480 & 541 & 345 & 368 & 335 & 341 & 345 & 362 \\
\hline Median rank & 11 & 12 & 11 & 36 & 16 & 20 & 12 & 10 & 9 & 12 & 16 & 14 \\
\hline
\end{tabular}


As we may observe, simple citations counting and in-degree perform best. This is not astonishing since prestige, popularity, awards, and recognition generally still rely mostly on the number of an individual's citations. What is more surprising is the very good result of HITS which is in contradiction with the conclusions taken by SIDIROPOULOS (2005). However, their HITS ranking was not obtained in the same way as ours.

\begin{tabular}{|lll}
$\rightarrow-$ C. Mohan & $\rightarrow$ David DeWitt & - David Maier \\
$\rightarrow$ Don Chamberlin & $\rightarrow-$ Hector Garcia-Molina & - - Jim Gray \\
$\rightarrow$ Michael Stonebraker & - Patricia Selinger & - Philip Bernstein \\
$\rightarrow$ Rakesh Agrawal & - Ronald Fagin & - Rudolf Bayer \\
$\square-$ Serge Abiteboul & - Jeffrey D. Ullman & - - Michael Carey \\
\hline
\end{tabular}

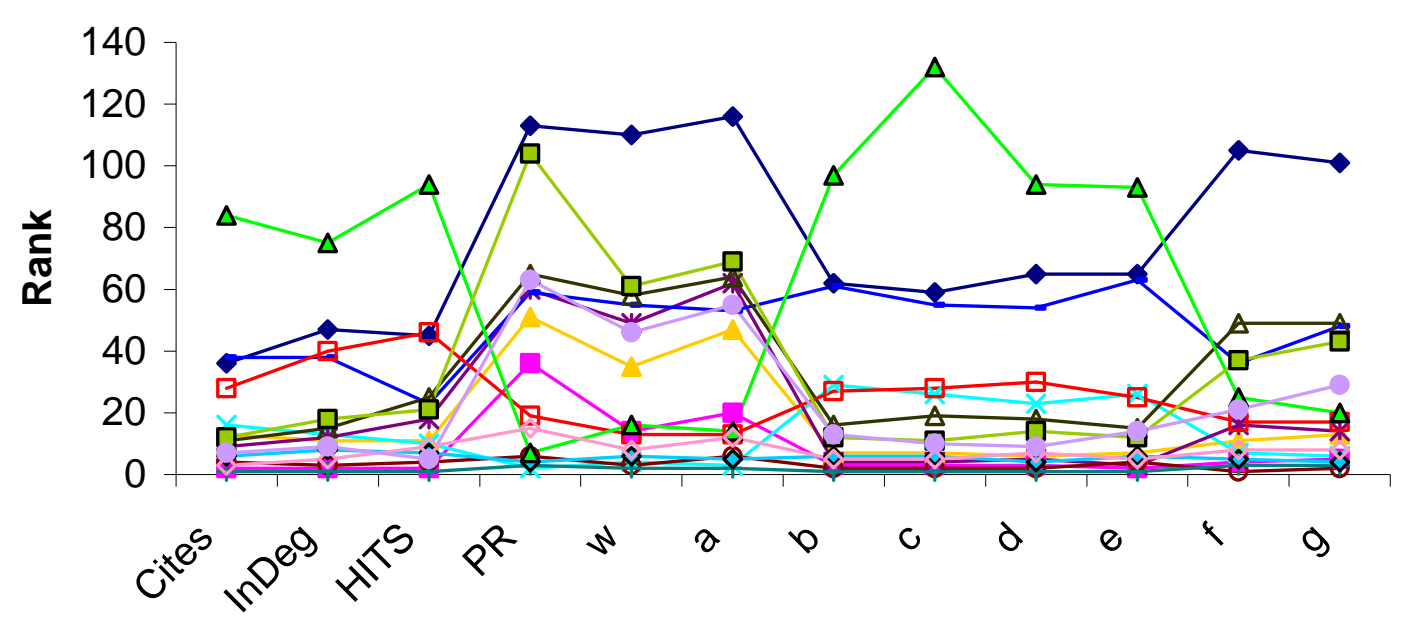

Ranking method

Figure 4: E. F. Codd Innovations Award winners and their ranks in distinct methods.

\section{Discussion of author ranks}

The accompanying chart of Table 5 is in Figure 4 . We can easily capture the most significant trends there. The three lowest-ever ranked authors are Rudolf Bayer, C. Mohan, and Serge Abiteboul. At the same time, the positions of Rudolf Bayer and Serge Abiteboul are quite oscillating (both high and low ranks exist) whereas those achieved by C. Mohan remain more stable (rather low). There are two scientists who are always 
ranked in the top 10 - Michael Stonebraker and Jim Gray. Nevertheless, these two researchers were awarded first - in 1992 and 1993, respectively. Thus, there has been time enough for them to profit from the award and to collect citations. In this context, the high ranks of the most recently awarded researcher, Jeffrey D. Ullman, are very remarkable. (Of course, he may have won another one from the many awards before.)

Let us have a look at some particularities in Figure 4. For instance, Rudolf Bayer has relatively few citations and few distinct citing authors (citations and in-degree), but he is cited mostly by authoritative researchers ("PR" and "w") and not so much by his colleagues ("a"). Then he suddenly looses good positions which may indicate that his colleagues citing him have published rather little ("b" and "e") and that they usually have few co-authors in their publications ("c" and "d"). But the number of co-authors in the common publications with the researchers citing him is relatively high ("f" and "g"). Also, there is the biggest difference between "c" and "d" for Rudolf Bayer amongst all awarded authors. This may mean that there are less distinct co-authors in his publications (and/or in publications of his colleagues citing him) with respect to all co-authors than is the case with other award winners. It is somewhat inverse with Serge Abiteboul. He has many citations but is cited by less authoritative authors (a sudden drop with "PR"). However, if the frequency of endorsements is taken into account (“w”), Abiteboul's rank improves considerably (from over 100 to almost 60), etc. Certainly, all of the above explanations are not exclusive, because there may be many other factors affecting the ranks that we are even not aware of. Also keep in mind that the results are based on the very incomplete data we work with. We do not present individual statistics over rankings for each author here since the objective is to compare rankings rather than authors. 


\section{Comparison of Rankings}

There are a number of metrics for comparison of rankings. See (SIDIROPOULOS, 2006) for some of them. We will briefly discuss the outcomes of three metrics - two numerical and one graphical. In Table 6 we can see the number of common elements in the top twenty authors of two particular rankings. For instance, the ranking by citations has 16 authors in common with the ranking by in-degree in the Top 20. The number of common authors varies between five and twenty. Of course, it does not reveal anything about the order of authors. It just says that 16 authors are the same. Theoretically, the ordering could be inverse. Two pairs of rankings have a complete match - "w" and "a", and "b" and "e". Also "f" and "g" have a rather great match (19 authors in common). On the other hand, the least observable match is produced by the standard PageRank - it shares just five authors with each "b", "c", and "e". We can notice that there is a set of pairs of "twin" rankings that match quite well

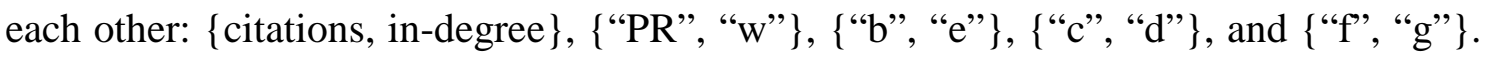
The "twin" rankings are very close to each other in the definition of their coefficients, e.g. weighted or unweighted in-degree, co-authors or distinct co-authors, etc. This definition similarity results in the similarity of their top twenty authors. The only exception in this respect is the pair $\{$ "w", "a"\} that matches perfectly but whose definition is somewhat distinct. On the contrary, we may observe the smallest numbers between the rankings from $\{$ “b”, “c”, “d”, "e" $\} X\{$ “PR", “w”, “a” $\}$.

The next comparison is based on the correlation between rankings. Table 7 shows the Spearman correlation coefficients for each pair of rankings. They are all significant at the 0.01 level. An alternative metric would be Kendall's tau. With this metric, we consider the ranks of all authors that have some in-degree. (It is 12934 as we 
mention above.) Thus, few matches in the Top 20 may be easily compensated for with matches of lower ranked researchers. All highly matching pairs of rankings from Table 6 are represented by a large correlation coefficient. The highest correlation $(0.9995)$ was measured between "b" and "e" where publications and "solo" publications are interchanged. On the other hand, the least correlation is reported between "c" and HITS (0.6379). However, the number of common top 20 authors is 12 which is by far not the worst. Evidently, there are many mismatches between lower-ranked scientists. The sector of small matches from Table 6 has disappeared here. It seems that mismatches just accumulate in the upper part of rankings (which is more important than the lower one, though).

Table 6: Common elements in top 20 authors of different rankings.

\begin{tabular}{|c|c|c|c|c|c|c|c|c|c|c|c|c|}
\hline & Cites & InDeg & HITS & PR & w & a & b & c & d & e & $f$ & g \\
\hline Cites & $x$ & 16 & 14 & 7 & 9 & 9 & 14 & 14 & 15 & 14 & 12 & 12 \\
\hline InDeg & 16 & $X$ & 16 & 9 & 10 & 10 & 12 & 12 & 13 & 12 & 13 & 13 \\
\hline HITS & 14 & 16 & $X$ & 11 & 12 & 12 & 11 & 12 & 13 & 11 & 16 & 15 \\
\hline PR & 7 & 9 & 11 & $X$ & 16 & 16 & 5 & 5 & 6 & 5 & 14 & 15 \\
\hline w & 9 & 10 & 12 & 16 & $X$ & 20 & 7 & 7 & 8 & 7 & 16 & 17 \\
\hline $\mathbf{a}$ & 9 & 10 & 12 & 16 & 20 & $X$ & 7 & 7 & 8 & 7 & 16 & 17 \\
\hline b & 14 & 12 & 11 & 5 & 7 & 7 & $X$ & 18 & 17 & 20 & 11 & 10 \\
\hline c & 14 & 12 & 12 & 5 & 7 & 7 & 18 & $x$ & 18 & 18 & 11 & 10 \\
\hline d & 15 & 13 & 13 & 6 & 8 & 8 & 17 & 18 & $x$ & 17 & 12 & 11 \\
\hline e & 14 & 12 & 11 & 5 & 7 & 7 & 20 & 18 & 17 & $x$ & 11 & 10 \\
\hline$f$ & 12 & 13 & 16 & 14 & 16 & 16 & 11 & 11 & 12 & 11 & $X$ & 19 \\
\hline $\mathbf{g}$ & 12 & 13 & 15 & 15 & 17 & 17 & 10 & 10 & 11 & 10 & 19 & $x$ \\
\hline
\end{tabular}

Table 7: Spearman correlation coefficients.

\begin{tabular}{|c|c|c|c|c|c|c|c|c|c|c|c|c|}
\hline & Cites & InDeg & HITS & PR & w & a & b & c & d & e & $f$ & g \\
\hline Cites & $x$ & 0.9904 & 0.8666 & 8119 & 8207 & 8188 & $81 \varepsilon$ & 807 & 815 & 32 & 82 & $82 \%$ \\
\hline Den & 0.9904 & $x$ & & & & & & & & & & 207 \\
\hline ITS & 0.8666 & 0.8661 & $X$ & 0.7748 & $0.74 \mathrm{~S}$ & 0.7483 & & & & & & 496 \\
\hline PR & 0.8119 & 0.8178 & 0.7748 & X & 0.9806 & 0.9803 & 0.9168 & 0.8 & 0.9 & & 30. & 10.9776 \\
\hline w & 0.8207 & 0.8179 & 0.7496 & 0.9806 & $x$ & 0.9993 & 0.9520 & 0.9197 & 0.95 & 0.9 & 60.9968 & 8 0.9981 \\
\hline a & 0.8188 & 0.816 & 0.7483 & 0.9803 & 0.9993 & $x$ & 0.9452 & 0.9123 & 0.9 & & 20.9938 & 80.9960 \\
\hline b & 0.8189 & 0.81 & $0.67 \varepsilon$ & 0.916 & 0.9520 & 0.9452 & $x$ & 0.9935 & 0.9992 & 0.95 & 50.9665 & 50.9620 \\
\hline c & 0.8079 & 0.8072 & 0.6379 & 0.8785 & 0.9197 & 0.9123 & 0.9935 & $x$ & 0.9921 & 0.9904 & 40.9376 & 60.9315 \\
\hline d & 0.8199 & 0.8178 & 0.6831 & 0.9213 & 0.9557 & 0.9491 & 0.9992 & 0.9921 & $X$ & 0.9993 & 30.9700 & 0.9657 \\
\hline e & 0.8203 & 0.8180 & 0.6866 & 0.9253 & 0.9586 & 0.9522 & 0.9995 & 0.9904 & 0.9993 & $3 x$ & 0.9722 & 20.9681 \\
\hline $\mathbf{f}$ & 0.8253 & 0.8221 & 0.7473 & 0.9751 & 0.9968 & 0.9938 & 0.9665 & 0.9376 & 0.9700 & 0.9722 & $2 \quad x$ & 0.9994 \\
\hline g & 0.8237 & 0.820 & 074 & 0.977 & 0.998 & 0.9960 & 0.9620 & 0.9315 & 0.96 & 0.9681 & 10.9994 & $4 \times$ \\
\hline
\end{tabular}


Finally, let us present a graphical representation called q-q plot. Ranks of authors generated by two different rankings are plotted against each other. Obviously, two perfectly matching rankings would produce a straight line. There are 68 ranking pairs, so it is impossible to show all charts. We have chosen four of them and show them in Figure 5. The top-left and bottom-left charts are examples of highly matching "twin" rankings ("f" vs. "g" and "b" vs. "e", respectively). The top-right plot is for the least correlating pair (HITS vs. "c") and the bottom-right plot represents a "mediocre" ranking pair (namely "a” vs. "c").
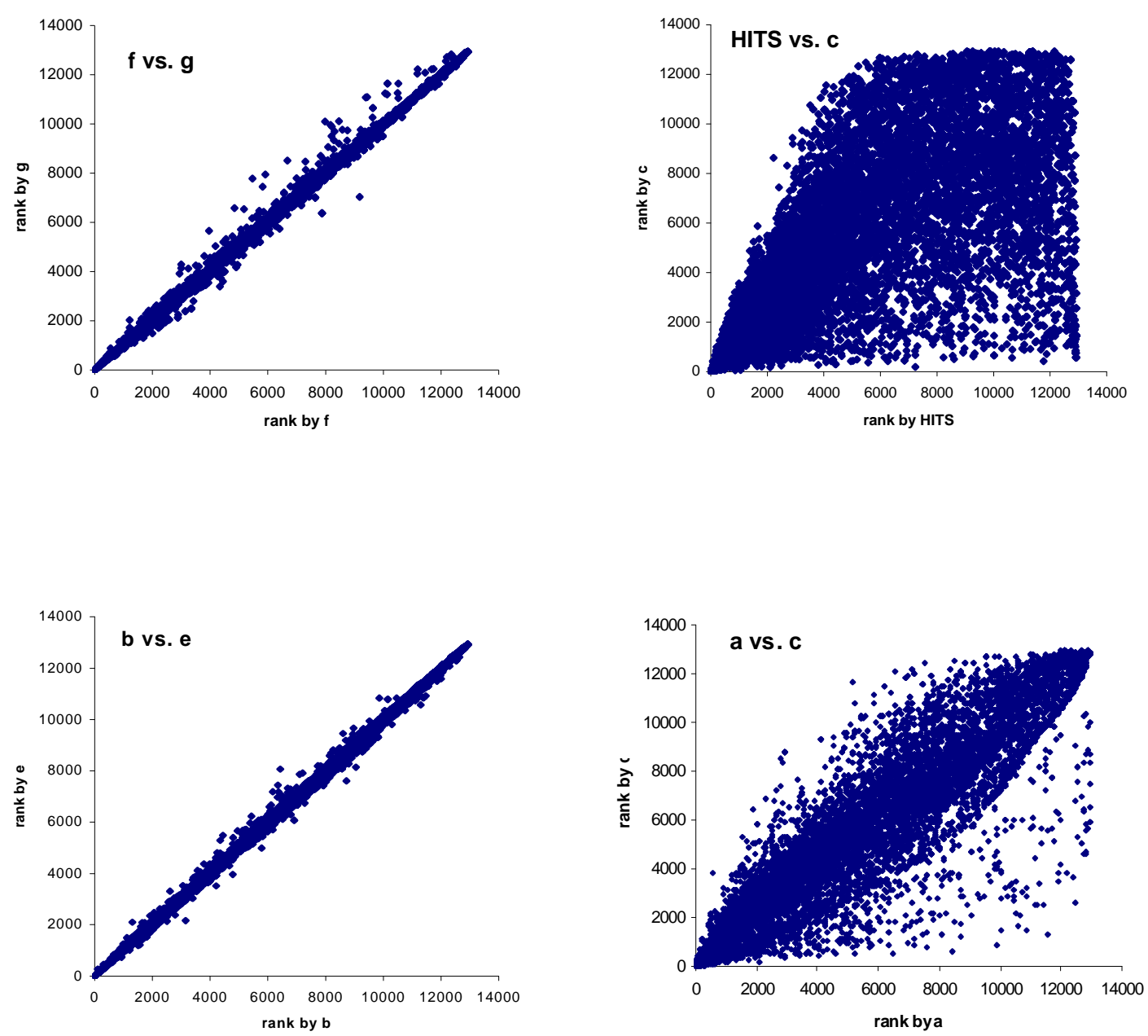

Figure 5: Some comparisons of rankings by means of q-q plots. 


\section{Convergence}

All in all, enhancing the citation graph with further bibliographic information proves to be very useful. The advantage over the standard PageRank is clear. Already assigning weights to the edges in the citation graph is very effective and adding data from the co-authorship network improves the results even more. The convergence rates of standard and bibliographic PageRanks are comparable. See Figure 6 where the damping factor ( $d$ in Equation 1$)$ is set to 0.9. The vertical axis in the figure represents the Spearman correlation coefficient between the rank vectors in the current and previous iteration. This simplified convergence criterion is often used instead of measuring the absolute error over rank scores. In the single precision arithmetic (six or seven decimal digits), all algorithms converge in about ten iterations. Of course, the resulting rankings depend entirely on the structure of the citation and co-authorship graphs, i.e. on the DBLP data they are generated from. In our data collection, only 8188 publications from the total 472043 had references included. The rest could be used for the co-authorship graph only. Even though the DBLP collection dates from 2004, it still makes sense to take into account award winners from more recent years because it usually takes a couple years for a publication to become cited and DBLP references to papers from years after 1997 are rather rare (SIDIROPOULOS, 2005). The newest citing paper is from 2001 as pointed out above.

\section{Prediction}

We show the top 40 authors for each ranking method in Table 8, Table 9, Table 10, and Table 11 in an appendix. E. F. Codd Award winners are in bold. Of course, the top ranked authors that have not yet been awarded have the greatest chance to win the award in future years. Raymond A. Lorie and Umeshwar Dayal appear 
among the best in each ranking. As the awarding highly correlates with the ranking by citations, Won Kim is also a top candidate for the ACM SIGMOD E. F. Innovations Award in future years. (E. F. Codd himself died in 2003 and cannot be awarded.)

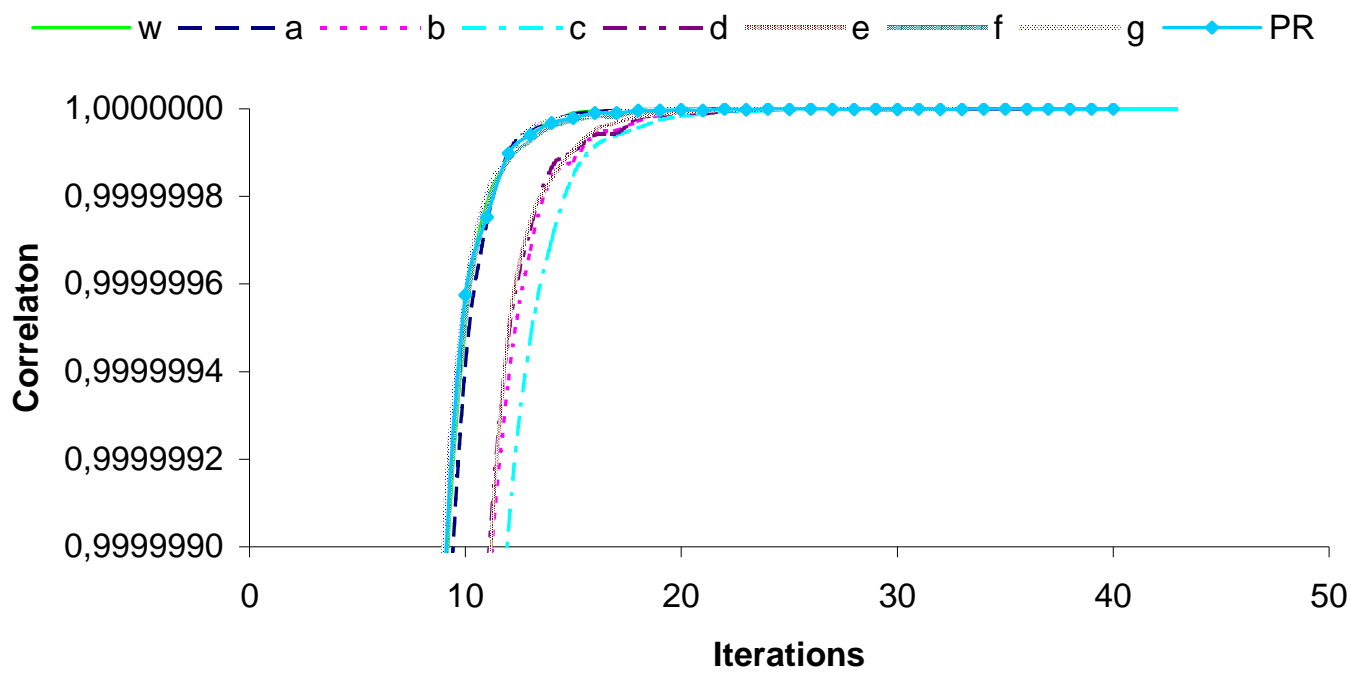

Figure 6: Convergence of standard (PR), weighted (w) and bibliographic (a-g) PR.

\section{Related Work}

\section{Sidiropoulos}

Sidiropoulos and Manolopoulos (SIDIROPOULOS, 2005) have proposed modifications of PageRank that would better meet needs for evaluating nodes in bibliographic networks. Their PageRank-based algorithm is called SCEAS. Although we adopted their testing methodology (DBLP and award winners) and tried our best for our results to be directly comparable, they are not. This has several reasons:

1. Different data. Unfortunately, authors use DBLP data from January 14, 2005. These data were probably up-to-date when they conducted their experiments but they are obsolete now and, in addition, they are not publicly available. Had they 
worked with the time-stamped data instead, the input data would be the same and their results verifiable.

2. No author citation graph. Only co-authorship graph $G^{P}$ and publication citation graph $G^{C}$ are constructed. All computations are performed upon $G^{C}$ and rankings for authors are obtained by averaging ranks of their publications.

3. Not all publications considered. In addition, only the ranks of the 25 best-ranked publications of each awarded author are counted in for author ranks. The number 25 was selected because it appeared to be the global optimum of SCEAS Rank.

Evidently, the number of best publications selected can severely affect the ranking quality. If a global optimum for PageRank was chosen instead, one can assume that SCEAS Rank would come out much worse. Even for those 25 publications (optimal for SCEAS), PageRank has a smaller sum of ranks (200 against 207). The results of SCEAS would be comparable to ours if the ranks of all publications for each author were taken into account. The authors do not disclose these results. Working directly at the author level (and not at the publication level) avoids the problem of searching for the optimal number of best publications for authors (some authors may even not have the required number of publications) and, therefore, the resulting rankings are biased towards the method that the optimal number of top publications was selected for. Authors in (SIDIROPOULOS, 2006) try to amend the "number-of-publications" problem by aggregating the ranks of authors over several different numbers of top publications but still not all publications are considered which does not allow for an unbiased comparison of authors and methods. The inherent disadvantage of our authorlevel methodology is that it does not enable ranking publications. 


\section{Liu and Bollen}

LIU (2005) introduces co-authorship frequency and exclusivity computed from a co-authorship graph into PageRank (called AuthorRank) and rank authors from a few conferences on digital libraries. Co-authorship frequency and exclusivity are somewhat analogous to the $c$ and $t$ coefficients from our definitions. Their testing data originating from an undisclosed version of DBLP are rather small (759 publications) and domainspecific. They compare their rankings with relevant program committee members and conclude that "the results of PageRank and AuthorRank are highly correlated, but there is no conclusive evidence that one performs better than the other." However, they do not take advantage of distinct numbers of citations between authors, i.e. the parameter $w$ from the definitions section is always set to one in their method. Interestingly, they do this for journal citation networks with a weighted PageRank algorithm (BOLLEN, 2006). But no co-authorship information was added to journals for obvious reasons. On the other hand, our "bibliographic" PageRank exploits both the co-authorship and citation information from bibliographic networks in a generalized manner.

\section{Conclusions}

Link-based ranking methods have become the standard way of determining authoritative Web pages. They may be easily applied in every environment that can be modelled as a graph and citation networks of authors or papers invite their usage. However, citation networks are only one part of bibliographic information. Collaboration networks are also a valuable source of information and their combination with citation graphs, which may lead to more "fair" rankings of authors, has been relatively little explored. Therefore, we present several modifications of the classical PageRank formula adapted for bibliographic networks. Our versions of PageRank take 
into account not only the citation but also the co-authorship graph. We verify the viability of our algorithms by applying them to the data from the DBLP digital library and by comparing the resulting ranks of the winners of the ACM SIGMOD Edgar F. Codd Innovations Award. Rankings based on both the citation and co-authorship information tend to place the awarded authors higher than the standard PageRank ranking. In our future work, we would like to concentrate on the issue of incorporating the time factor in the bibliographic PageRank.

This work was supported in part by the Ministry of Education of the Czech Republic under Grant 2C06009.

\section{References}

BALMIN, A., HRISTIDIS, V., PAPAKONSTANTINOU, Y. (2004), ObjectRank: Authority-Based Keyword Search in Databases, Proc. 30th Int. Conf. Very Large Data Bases, Toronto, Canada, pp. 564-575.

BIANCHINI, M., GORI, M., SCARSELLI, F. (2005), Inside PageRank, ACM Transactions on Internet Technology, 5(1):92-128.

BOLLEN, J., RODRIQUEZ, M. A., VAN DE SOMPEL, H. (2006), Journal status, Scientometrics, 69(3):669-687.

BRIN, S., PAGE, L. (1998), The Anatomy of a Large-Scale Hypertextual Web Search Engine, Proc. 7th World Wide Web Conference, pp. $107-117$.

CUNNINGHAM, S. J., DILLON, S. M. (1997), Authorship patterns in information systems, Scientometrics, 39(1):19-27.

ELMACIOGLU, E., LEE, D. (2005), On six degrees of separation in DBLP-DB and more, SIGMOD Record, 34(2):33-40. 
LANGVILlE, A. N., MEYER, C. D. (2003), Deeper Inside PageRank, Internet Mathematics, 1(3):335-380.

LIU, X., BOLLEN, J., NELSON, M. L., VAN DE SOMPEL, H. (2005), Co-authorship Networks in the Digital Library Research Community, Information Processing and Management, 41(6):1462-1480.

NASCIMENTO, M. A., SANDER, J., POUND, J. (2003), Analysis of SIGMOD's coauthorship graph, SIGMOD Record, 32(3):8-10.

OTTE E., ROUSSEAU R. (2002), Social network analysis: a powerful strategy, also for the information sciences, Journal of Information Science, 28(6):441-453.

RAHM, E., THOR, A. (2005), Citation analysis of database publications, SIGMOD Record, 34(4):48-53.

SIDIROPOUlOS, A., MANOLOPOUlOS, Y. (2005), A Citation-Based System to Assist Prize Awarding, SIGMOD Record, 34(4):54-60.

SIDIROPOULOS, A., MANOLOPOULOS, Y. (2006), A Generalized comparison of graph-based ranking algorithms for publications and authors, Journal of Systems and Software, 79(12):1679-1700.

WAGNER, C., LEYDESDORFF, L. (2003), Mapping global science using international co-authorships: A comparison of 1990 and 2000, Proc. 9th Int. Conf. Scientometrics and Informetrics, Dalian, China, pp. 330-340.

XING, W., GHORBANI, A. (2004), Weighted PageRank algorithm, Proc. 2nd Conf. Communication Networks and Services Research, Fredericton, Canada, pp. 305-314. 


\section{Appendix}

Table 8: Top 40 DBLP authors for each ranking (part 1).

\begin{tabular}{|c|c|c|c|c|}
\hline Citations & & In-degree & & HITS \\
\hline 1 Michael Stonebraker & 5346 & Michael Stonebraker & 1857 & Michael Stonebraker \\
\hline 2 David J. Dewitt & 4865 & David J. Dewitt & 1432 & David J. Dewitt \\
\hline 3 Jeffrey D. UlIman & 3926 & Jim Gray & 1347 & Raymond A. Lorie \\
\hline 4 Jim Gray & 3702 & Raymond A. Lorie & 1250 & Jim Gray \\
\hline 5 Raymond A. Lorie & 3317 & Jeffrey D. Ullman & 1156 & Michael J. Carey \\
\hline 6 Philip A. Bernstein & 2893 & Won Kim & 1113 & Won Kim \\
\hline 7 Michael J. Carey & 2773 & E. F. Codd & 1110 & Philip A. Bernstein \\
\hline 8 E. F. Codd & 2732 & Philip A. Bernstein & 1109 & Umeshwar Dayal \\
\hline 9 Hector Garcia-Molina & 2696 & Michael J. Carey & 1042 & Jeffrey D. UlIman \\
\hline 10 Won Kim & 2670 & Umeshwar Dayal & 1035 & Donald D. Chamberlin \\
\hline 11 Rakesh Agrawal & 2640 & David Maier & 983 & David Maier \\
\hline 12 Serge Abiteboul & 2601 & Hector Garcia-Molina & 974 & Morton M. Astrahan \\
\hline 13 David Maier & 2448 & Donald D. Chamberlin & 940 & François Bancilhon \\
\hline 14 Umeshwar Dayal & 2301 & Peter P. Chen & 896 & Bruce G. Lindsay \\
\hline 15 Yehoshua Sagiv & 2160 & Rakesh Agrawal & 855 & Kapali P. Eswaran \\
\hline 16 Donald D. Chamberlin & 2099 & Morton M. Astrahan & 829 & Hamid Pirahesh \\
\hline 17 Catriel Beeri & 2089 & Kapali P. Eswaran & 820 & E. F. Codd \\
\hline 18 François Bancilhon & 2059 & Serge Abiteboul & 809 & Hector Garcia-Molina \\
\hline 19 Christos Faloutsos & 1970 & Nathan Goodman & 804 & Eugene Wong \\
\hline 20 Jennifer Widom & 1937 & François Bancilhon & 802 & Irving L. Traiger \\
\hline 21 Nathan Goodman & 1928 & Hamid Pirahesh & 765 & Serge Abiteboul \\
\hline 22 Morton M. Astrahan & 1847 & Bruce G. Lindsay & 761 & Nathan Goodman \\
\hline 23 Raghu Ramakrishnan & 1825 & Irving L. Traiger & 760 & Patricia G. Selinger \\
\hline 24 Irving L. Traiger & 1708 & Eugene Wong & 742 & Thomas G. Price \\
\hline 25 Jeffrey F. Naughton & 1704 & Catriel Beeri & 709 & Rakesh Agrawal \\
\hline 26 Eugene Wong & 1600 & Jennifer Widom & 696 & Catriel Beeri \\
\hline 27 Hamid Pirahesh & 1600 & Randy H. Katz & 676 & Patrick Valduriez \\
\hline 28 Ronald Fagin & 1599 & Jeffrey F. Naughton & 675 & Stanley B. Zdonik \\
\hline 29 Kapali P. Eswaran & 1595 & Nick Roussopoulos & 674 & Yehoshua Sagiv \\
\hline 30 Bruce G. Lindsay & 1548 & Stanley B. Zdonik & 670 & Lawrence A. Rowe \\
\hline 31 Peter P. Chen & 1511 & Raghu Ramakrishnan & 667 & Jeffrey F. Naughton \\
\hline 32 Richard Hull & 1488 & Yehoshua Sagiv & 661 & Randy H. Katz \\
\hline 33 Nick Roussopoulos & 1383 & Shamkant B. Navathe & 650 & Jennifer Widom \\
\hline 34 Randy H. Katz & 1381 & John Miles Smith & 645 & Raghu Ramakrishnan \\
\hline 35 Patrick Valduriez & 1373 & H. V. Jagadish & 640 & Nick Roussopoulos \\
\hline 36 C. Mohan & 1350 & Patrick Valduriez & 621 & Carlo Zaniolo \\
\hline 37 H. V. Jagadish & 1343 & Henry F. Korth & 619 & Henry F. Korth \\
\hline 38 Patricia G. Selinger & 1341 & Patricia G. Selinger & 619 & Mike W. Blasgen \\
\hline 39 Stanley B. Zdonik & 1336 & Thomas G. Price & 616 & Goetz Graefe \\
\hline 40 Goetz Graefe & 1327 & Ronald Fagin & 613 & Gianfranco R. Putzolu \\
\hline \multicolumn{2}{|c|}{ Missed: 84. Rudolf Bayer (845) } & \multicolumn{2}{|c|}{$\begin{array}{l}\text { Missed: 47. C. Mohan (578), } 75 . \\
\text { Rudolf Bayer (466) }\end{array}$} & $\begin{array}{l}\text { Missed: 45. C. Mohan, } \\
\text { 46. Ronald Fagin, } 94 . \\
\text { Rudolf Bayer }\end{array}$ \\
\hline
\end{tabular}


Table 9: Top 40 DBLP authors for each ranking (part 2).

\begin{tabular}{|c|c|c|c|}
\hline & PR & $\mathbf{w}$ & $\mathbf{a}$ \\
\hline 1 & E. F. Codd & E. F. Codd & E. F. Codd \\
\hline 2 & Donald D. Chamberlin & Michael Stonebraker & Michael Stonebraker \\
\hline 3 & Michael Stonebraker & Jim Gray & Donald D. Chamberlin \\
\hline 4 & Philip A. Bernstein & Donald D. Chamberlin & Raymond A. Lorie \\
\hline 5 & John Miles Smith & Raymond A. Lorie & Philip A. Bernstein \\
\hline 6 & Jim Gray & Philip A. Bernstein & Jim Gray \\
\hline 7 & Rudolf Bayer & John Miles Smith & John Miles Smith \\
\hline 8 & Raymond A. Lorie & Jeffrey D. UlIman & Morton M. Astrahan \\
\hline 9 & Morton M. Astrahan & Morton M. Astrahan & Irving L. Traiger \\
\hline 10 & Kapali P. Eswaran & Irving L. Traiger & Eugene Wong \\
\hline 11 & Eugene Wong & Eugene Wong & Kapali P. Eswaran \\
\hline 12 & Irving L. Traiger & Kapali P. Eswaran & Jeffrey D. UlIman \\
\hline 13 & Gerald Held & Ronald Fagin & Ronald Fagin \\
\hline 14 & Hans Albrecht Schmid & David J. Dewitt & Rudolf Bayer \\
\hline 15 & Jeffrey D. UlIman & Catriel Beeri & Catriel Beeri \\
\hline 16 & Michael Hammer & Rudolf Bayer & William C. McGee \\
\hline 17 & Mike W. Blasgen & William C. McGee & Gerald Held \\
\hline 18 & Raymond F. Boyce & Gerald Held & Diane C. P. Smith \\
\hline 19 & Ronald Fagin & Gianfranco R. Putzolu & Gianfranco R. Putzolu \\
\hline 20 & Gianfranco R. Putzolu & Diane C. P. Smith & David J. Dewitt \\
\hline 21 & Edward M. McCreight & Nathan Goodman & Nathan Goodman \\
\hline 22 & Nathan Goodman & Michael Hammer & Michael Hammer \\
\hline 23 & James W. Mehl & Mike W. Blasgen & Mike W. Blasgen \\
\hline 24 & W. Frank King III & Stephen Todd & Hans Albrecht Schmid \\
\hline 25 & Bradford W. Wade & Hans Albrecht Schmid & Stephen Todd \\
\hline 26 & Paul R. McJones & Bradford W. Wade & Paul R. McJones \\
\hline 27 & Robert C. Goldstein & James W. Mehl & Bradford W. Wade \\
\hline 28 & Stephen Todd & Paul R. McJones & James W. Mehl \\
\hline 29 & Patricia P. Griffiths & W. Frank King III & Patricia P. Griffiths \\
\hline 30 & Diane C. P. Smith & Patricia P. Griffiths & W. Frank King III \\
\hline 31 & Philip Yen-tang Chang & Alfred V. Aho & Alfred V. Aho \\
\hline 32 & Peter Kreps & Peter Kreps & Peter Kreps \\
\hline 33 & Vera Watson & Yehoshua Sagiv & Edward M. McCreight \\
\hline 34 & Peter P. Chen & Edward M. McCreight & Robert C. Goldstein \\
\hline 35 & Catriel Beeri & David Maier & Moshé M. Zloof \\
\hline 36 & David J. Dewitt & Robert C. Goldstein & Philip Yen-tang Chang \\
\hline 37 & Alfred V. Aho & Raymond F. Boyce & Raymond F. Boyce \\
\hline 38 & John J. Donovan & Moshé M. Zloof & Vera Watson \\
\hline 39 & Stuart G. Greenberg & Vera Watson & C. J. Date \\
\hline 40 & Loius M. Gutentag & Umeshwar Dayal & Peter P. Chen \\
\hline & $\begin{array}{l}\text { Missed: 51. David Maier, } 59 . \\
\text { Patricia Selinger, 60. Hector } \\
\text { Garcia-Molina, 63. Michael } \\
\text { Carey, 65. Rakesh Agrawal, } \\
\text { 104. Serge Abiteboul, } 113 . \\
\text { C. Mohan }\end{array}$ & $\begin{array}{l}\text { Missed: 46. Michael Carey, } \\
\text { 49. Hector Garcia-Molina, } 55 . \\
\text { Patricia Selinger, 58. Rakesh } \\
\text { Agrawal, 61. Serge } \\
\text { Abiteboul, 110. C. Mohan }\end{array}$ & $\begin{array}{l}\text { Missed: 47. David Maier, } 53 . \\
\text { Patricia Selinger, 55. Michael } \\
\text { Carey, 62. Hector Garcia- } \\
\text { Molina, 64. Rakesh Agrawal, } \\
\text { 69. Serge Abiteboul, 116. C. } \\
\text { Mohan }\end{array}$ \\
\hline
\end{tabular}


Table 10: Top 40 DBLP authors for each ranking (part 3).

\begin{tabular}{|c|c|c|}
\hline b & c & d \\
\hline 1 Michael Stonebraker & Michael Stonebraker & Michael Stonebraker \\
\hline 2 Jim Gray & Jim Gray & Jim Gray \\
\hline 3 David J. Dewitt & David J. Dewitt & David J. Dewitt \\
\hline 4 Hector Garcia-Molina & Hector Garcia-Molina & Philip A. Bernstein \\
\hline 5 Jeffrey D. UlIman & Jeffrey D. UlIman & Hector Garcia-Molina \\
\hline 6 Philip A. Bernstein & Philip A. Bernstein & David Maier \\
\hline 7 David Maier & David Maier & Jeffrey D. Ullman \\
\hline 8 Moshe Y. Vardi & Umeshwar Dayal & Umeshwar Dayal \\
\hline 9 E. F. Codd & Bruce G. Lindsay & Michael J. Carey \\
\hline 10 Catriel Beeri & Michael J. Carey & E. F. Codd \\
\hline 11 Umeshwar Dayal & Serge Abiteboul & Bruce G. Lindsay \\
\hline 12 Serge Abiteboul & Jeffrey F. Naughton & Catriel Beeri \\
\hline 13 Michael J. Carey & Catriel Beeri & Jeffrey F. Naughton \\
\hline 14 Yehoshua Sagiv & Hamid Pirahesh & Serge Abiteboul \\
\hline 15 Christos H. Papadimitriou & Moshe Y. Vardi & Hamid Pirahesh \\
\hline 16 Rakesh Agrawal & Hans-Jörg Schek & Goetz Graefe \\
\hline 17 Bruce G. Lindsay & E. F. Codd & Hans-Jörg Schek \\
\hline 18 Jeffrey F. Naughton & Yehoshua Sagiv & Rakesh Agrawal \\
\hline 19 Nick Roussopoulos & Rakesh Agrawal & Raymond A. Lorie \\
\hline 20 Hans-Jörg Schek & Raghu Ramakrishnan & Yehoshua Sagiv \\
\hline 21 Raghu Ramakrishnan & Goetz Graefe & Nick Roussopoulos \\
\hline 22 Hamid Pirahesh & Nick Roussopoulos & Gio Wiederhold \\
\hline 23 Goetz Graefe & Raymond A. Lorie & Donald D. Chamberlin \\
\hline 24 Raymond A. Lorie & Christos H. Papadimitriou & Moshe Y. Vardi \\
\hline 25 Alberto O. Mendelzon & Gio Wiederhold & Dina Bitton \\
\hline 26 Gio Wiederhold & Donald D. Chamberlin & Richard T. Snodgrass \\
\hline 27 Ronald Fagin & Richard T. Snodgrass & Christos H. Papadimitriou \\
\hline 28 Richard T. Snodgrass & Ronald Fagin & Raghu Ramakrishnan \\
\hline 29 Donald D. Chamberlin & Dina Bitton & Guy M. Lohman \\
\hline 30 François Bancilhon & Jennifer Widom & Ronald Fagin \\
\hline 31 Mihalis Yannakakis & Randy H. Katz & Randy H. Katz \\
\hline 32 Jennifer Widom & Alberto O. Mendelzon & François Bancilhon \\
\hline 33 Nathan Goodman & Guy M. Lohman & Alberto O. Mendelzon \\
\hline 34 Randy H. Katz & François Bancilhon & Jennifer Widom \\
\hline 35 H. V. Jagadish & H. V. Jagadish & Michael J. Franklin \\
\hline 36 Won Kim & Abraham Silberschatz & Irving L. Traiger \\
\hline 37 Irving L. Traiger & Irving L. Traiger & H. V. Jagadish \\
\hline 38 Abraham Silberschatz & Michael J. Franklin & Won Kim \\
\hline 39 Eugene Wong & Mihalis Yannakakis & Eugene Wong \\
\hline 40 Guy M. Lohman & Nathan Goodman & Nathan Goodman \\
\hline $\begin{array}{l}\text { Missed: 61. Patricia } \\
\text { Selinger, 62. C. Mohan, } 97 . \\
\text { Rudolf Bayer }\end{array}$ & $\begin{array}{l}\text { Missed: 55. Patricia Selinger, } \\
\text { 59. C. Mohan, 132. Rudolf } \\
\text { Bayer }\end{array}$ & $\begin{array}{l}\text { Missed: 54. Patricia Selinger, } \\
\text { 65. C. Mohan, 94. Rudolf } \\
\text { Bayer }\end{array}$ \\
\hline
\end{tabular}


Table 11: Top 40 DBLP authors for each ranking (part 4).

\begin{tabular}{|c|c|c|}
\hline e & $f$ & g \\
\hline 1 Michael Stonebraker & Jim Gray & E. F. Codd \\
\hline 2 David J. Dewitt & E. F. Codd & Jim Gray \\
\hline 3 Hector Garcia-Molina & Michael Stonebraker & Michael Stonebraker \\
\hline 4 Jim Gray & David J. Dewitt & Philip A. Bernstein \\
\hline 5 Jeffrey D. Ullman & Philip A. Bernstein & David J. Dewitt \\
\hline 6 Philip A. Bernstein & Raymond A. Lorie & Donald D. Chamberlin \\
\hline 7 David Maier & Donald D. Chamberlin & Raymond A. Lorie \\
\hline 8 Moshe Y. Vardi & Jeffrey D. Ullman & Jeffrey D. Ullman \\
\hline 9 Umeshwar Dayal & Irving L. Traiger & Irving L. Traiger \\
\hline 10 Catriel Beeri & Morton M. Astrahan & Morton M. Astrahan \\
\hline 11 E. F. Codd & David Maier & John Miles Smith \\
\hline 12 Serge Abiteboul & Eugene Wong & Eugene Wong \\
\hline 13 Yehoshua Sagiv & Catriel Beeri & David Maier \\
\hline 14 Michael J. Carey & John Miles Smith & Hector Garcia-Molina \\
\hline 15 Rakesh Agrawal & Bruce G. Lindsay & Catriel Beeri \\
\hline 16 Christos H. Papadimitriou & Hector Garcia-Molina & Kapali P. Eswaran \\
\hline 17 Bruce G. Lindsay & Ronald Fagin & Ronald Fagin \\
\hline 18 Jeffrey F. Naughton & Kapali P. Eswaran & Gerald Held \\
\hline 19 Nick Roussopoulos & Gerald Held & Umeshwar Dayal \\
\hline 20 Hans-Jörg Schek & Umeshwar Dayal & Rudolf Bayer \\
\hline 21 Raghu Ramakrishnan & Michael J. Carey & Michael Hammer \\
\hline 22 Hamid Pirahesh & Yehoshua Sagiv & Bruce G. Lindsay \\
\hline 23 Raymond A. Lorie & Gianfranco R. Putzolu & Nathan Goodman \\
\hline 24 Alberto O. Mendelzon & Nathan Goodman & Gianfranco R. Putzolu \\
\hline 25 Ronald Fagin & Rudolf Bayer & Stephen Todd \\
\hline 26 Donald D. Chamberlin & Mike W. Blasgen & Diane C. P. Smith \\
\hline 27 Gio Wiederhold & Michael Hammer & William C. McGee \\
\hline 28 Goetz Graefe & William C. McGee & Mike W. Blasgen \\
\hline 29 Nathan Goodman & Stephen Todd & Michael J. Carey \\
\hline 30 Mihalis Yannakakis & Diane C. P. Smith & Phyllis Reisner \\
\hline 31 François Bancilhon & Jeffrey F. Naughton & Paul R. McJones \\
\hline 32 Jennifer Widom & Thomas G. Price & Jeffrey F. Naughton \\
\hline 33 Randy H. Katz & Bradford W. Wade & Hamid Pirahesh \\
\hline 34 Richard T. Snodgrass & Hamid Pirahesh & Yehoshua Sagiv \\
\hline 35 Abraham Silberschatz & Phyllis Reisner & Bradford W. Wade \\
\hline 36 H. V. Jagadish & Patricia G. Selinger & Hans Albrecht Schmid \\
\hline 37 Guy M. Lohman & Serge Abiteboul & Nick Roussopoulos \\
\hline 38 Eugene Wong & W. Frank King III & Won Kim \\
\hline 39 Peter Buneman & François Bancilhon & James W. Mehl \\
\hline 40 Christos Faloutsos & James W. Mehl & W. Frank King III \\
\hline $\begin{array}{l}\text { Missed: 63. Patricia Selinger, } \\
\text { 65. C. Mohan, 93. Rudolf } \\
\text { Bayer }\end{array}$ & $\begin{array}{l}\text { Missed: 49. Rakesh Agrawal, } \\
\text { 105. C. Mohan }\end{array}$ & $\begin{array}{l}\text { Missed: 43. Serge Abiteboul, } \\
\text { 48. Patricia Selinger, } 49 . \\
\text { Rakesh Agrawal, 101. C. } \\
\text { Mohan }\end{array}$ \\
\hline
\end{tabular}

